\title{
Numerical Applicability of Different Sound Source Formulations to Compute Combustion Noise Using Acoustic Perturbation Equations for Reacting Flows
}

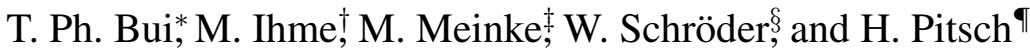 \\ RWTH Aachen University, Aachen, Germany \\ University of Michigan, Ann Arbor, MI 48109, USA \\ Stanford University, Stanford, CA 94305, USA
}

\begin{abstract}
Combustion noise analyses in a hybrid computational aeroacoustics (CAA) context are presented. Acoustic perturbation equations for reacting flows (APE-RF) describe the wave propagation, while unsteady results from a variable density incompressible reactive large-eddy simulation (LES) are used to evaluate the acoustic source terms. To simulate combustion generated noise via such a hybrid approach, an appropriate source description has to be taken, which preferably matches two requirements, i.e., on the one hand, to efficiently and accurately predict the generated acoustic field, and on the other hand, to easily determine the source term from the LES. In this study, three source formulations for the APE-RF system are examined on two acoustic meshes with different resolutions in the source region to predict the acoustic field of an open turbulent nonpremixed flame. Using the source term, which is expressed via the scaled partial time derivative of the density, the acoustic field can be reproduced best up to a maximum Strouhal number of $S t_{D}=2$ on the fine mesh. However, for this source formulation spurious noise can be observed depending on the CAA resolution. It will be shown in this study that this observation can be related to the "artificial interpolation induced acceleration" effect. A compromise between efficiency and accuracy represents the source formulation expressed via the scaled material derivative of the density.
\end{abstract}

\section{Introduction}

The main focus of this paper is to derive and to investigate suitable source formulations to be used in a hybrid CFD/CAA method to predict combustion generated noise. Therefore, the numerical applicability of three different sound source formulations in conjunction with the acoustic perturbation equations for reacting flows (APE-RF) is investigated. This study extends the previous investigation by Bui et al., ${ }^{1}$ in which the three source formulations for the APE-RF system have been derived based on the inclusion of the major sound generating mechanism, i.e., the unsteady heat release rate. The sound sources of interest are evaluated from a variable density incompressible reactive large-eddy simulation (LES). Among these three options, the results ${ }^{1}$ show the substantial time derivative of the density to be the most appropriate source formulation. Nevertheless, the source expressed via the partial time derivative of the density includes most source mechanisms and shows the smoothest spatial distribution compared to the other formulations. In other words, for these two reasons, this formulation was expected to yield the best acoustic result. However, the numerical findings did not verify the expectations Moreover, the spectra obtained from the simulation using the unsteady heat release as the major source term show an unexpected behavior. This latter source is deemed the major sound source in low MACH number flows, which is not entirely supported by the numerical results in the frequency range up to $S t_{D} \leq 2$.

To improve the understanding of the numerical results of the previous study, the sources of the acoustic perturbation equations for reacting flows (APE-RF) are interpolated onto two CAA grids with different resolutions. A coarse

\footnotetext{
* Research Associate, RWTH Aachen University, (p.bui@aia.rwth-aachen.de)

$\dagger$ Assistant Professor, University of Michigan, (mihme@umich.edu)

${ }^{\ddagger}$ Senior Scientist, RWTH Aachen University

§ Professor, RWTH Aachen University

ॠAssistant Professor, Stanford University
} 
and a very fine mesh are generated to study the impact of source term interpolation and the numerical applicability of the aforementioned sound source formulations in terms of numerical accuracy and efficiency. Note, if the interpolation error of an underresolved source term is accompanied by high frequency oscillations in the acoustic signal and additionall is misinterpreted by the numerical approach of the system governing the excitation and propagation of the acoustic waves, considerable spurious noise can occur. That is, the convection speed of density inhomogeneities cannot be preserved during interpolation, the effect of "artificial interpolation induced acceleration" will be shown to be a cause of spurious noise production.

Since the governing equations of the flow problem are given in Bui et al. ${ }^{1}$ this paper focuses on the following contents. First, the APE-RF system plus sources is briefly summarized. The three different source formulations and the source mechanisms inherently contained in each expression are specified. In the second part, the source term distribution is described and discussed followed by a comparison of the numerical results with experimental data. Acoustic simulations are performed on two grids with different resolutions, especially in the source domain. Special emphasis is placed on the analysis of the applicability of each source formulation with respect to the numerical efficiency and accuracy. The influence of source term interpolation in conjunction with the different APE-RF source formulations is discussed, followed by a presentation of a test case to evidence the occurrence of a spurious effect, namely the "artificial interpolation induced acceleration". Subsequently, the results are discussed with respect to the acoustic impact as to the spectral content and the directivity behavior is analyzed. Finally, the results are summarized, followed by some concluding remarks.

\section{Simplified APE-RF source formulations}

The APE-RF system plus all right-hand side (RHS) sources are presented subsequently. This system result from rearranging the conservation equations governing multi-component reacting flows such that the left-hand side constitutes the original homogeneous APE system, ${ }^{2}$ while the remaining terms are shifted to the RHS. This system reads

$$
\begin{aligned}
\frac{\partial \rho^{\prime}}{\partial t}+\nabla \cdot\left(\rho^{\prime} \overline{\boldsymbol{u}}+\bar{\rho} \boldsymbol{u}^{\prime}\right) & =q_{c, r f} \\
\frac{\partial \boldsymbol{u}^{\prime}}{\partial t}+\nabla\left(\overline{\boldsymbol{u}} \cdot \boldsymbol{u}^{\prime}\right)+\nabla\left(\frac{p^{\prime}}{\bar{\rho}}\right) & =\boldsymbol{q}_{\boldsymbol{m}, \boldsymbol{r} \boldsymbol{f}} \\
\frac{\partial p^{\prime}}{\partial t}-\bar{c}^{2} \frac{\partial \rho^{\prime}}{\partial t} & =q_{e, r f}
\end{aligned}
$$

with the right-hand side sources

$$
\begin{aligned}
q_{c, r f}= & -\nabla \cdot\left(\rho^{\prime} \boldsymbol{u}^{\prime}\right)^{\prime} \\
\boldsymbol{q}_{\boldsymbol{m}, \boldsymbol{r} \boldsymbol{f}}= & \nabla p\left(\frac{1}{\bar{\rho}}-\frac{1}{\rho}\right)-\frac{1}{\bar{\rho}}\left[\nabla \bar{p}+\left(\frac{\rho}{\bar{\rho}} c^{2}-\bar{c}^{2}\right) \nabla \bar{\rho}\right]+\left[\frac{\nabla \cdot \boldsymbol{\tau}}{\rho}+\sum_{n} Y_{n} \boldsymbol{f}_{\boldsymbol{n}}\right. \\
& \left.-(\overline{\boldsymbol{u}} \cdot \nabla) \overline{\boldsymbol{u}}-\left(\boldsymbol{u}^{\prime} \cdot \nabla\right) \boldsymbol{u}^{\prime}-\left(\boldsymbol{\omega}^{\prime} \times \overline{\boldsymbol{u}}\right)-\left(\overline{\boldsymbol{\omega}} \times \boldsymbol{u}^{\prime}\right)\right] \\
q_{e, r f}= & -\bar{c}^{2}\left[( \frac { \overline { \rho } } { \rho } + \frac { p - \overline { p } } { \rho \overline { c } ^ { 2 } } ) \left\{\frac{1}{c^{2}} \frac{D p}{D t}+\frac{\alpha}{c_{p}}\left(S_{I A}+S_{I B}+S_{I C}+\nabla \cdot \boldsymbol{q}\right.\right.\right. \\
& \left.\left.\left.-\frac{\partial u_{i}}{\partial x_{j}} \tau_{i j}\right)\right\}-\nabla \cdot\left(\boldsymbol{u} \rho_{e}\right)-\boldsymbol{u} \cdot \nabla \bar{\rho}-\frac{D}{D t}\left(\frac{p-\bar{p}}{\bar{c}^{2}}\right)\right] .
\end{aligned}
$$

Perturbation and time averaged quantities are denoted by a prime and an overbar, respectively. The thermoacoustic sources $S_{I A}, S_{I B}$, and $S_{I C}$ are defined as

$$
\begin{aligned}
\left.\sum_{n=1}^{N} \frac{\partial h}{\partial Y_{n}}\right|_{\rho, p, Y_{m}} \rho \frac{D Y_{n}}{D t}= & \underbrace{\left.\sum_{n=1}^{N} \frac{\partial h}{\partial Y_{n}}\right|_{T, p, Y_{m}} \omega_{n}}_{S_{I A}}-\underbrace{\left.\frac{\partial h}{\partial \rho}\right|_{p, Y_{n}}\left(\left.\sum_{n=1}^{N} \frac{\partial \rho}{\partial Y_{n}}\right|_{T, p, Y_{m}} \omega_{n}\right)}_{S_{I B}} \\
& -\underbrace{\left.\sum_{n=1}^{N} \frac{\partial h}{\partial Y_{n}}\right|_{\rho, p, Y_{m}} \nabla \cdot \boldsymbol{J}_{n}}_{S_{I C}} .
\end{aligned}
$$


They represent the effect due to unsteady heat release, non-isomolar combustion, and species diffusion. In addition to these source mechanisms, the APE-RF pressure-density relation includes source terms describing the effect of heat flux, viscosity, pressure fluctuations, acceleration of density inhomogeneities $\left(\nabla \cdot\left(\boldsymbol{u} \rho_{e}\right)\right)$, and non-uniform mean flows. For combustion noise simulations this homogeneous system has been chosen to take advantage of its benign properties to simulate wave propagation, i.e., its validity for irrotational non-uniform mean flows, while excitations of instabilities are prevented. It has to be emphasized that during the derivation of the APE-RF system no assumptions about nonpremixed or premixed flames have been made, such that the APE-RF system is generally valid for multi-component reacting flows. A detailed derivation of the APE-RF system has been published by Bui et al. ${ }^{3}$

Two simplified source formulations in conjunction with the APE-RF system has been derived, ${ }^{4}$ to simulate combustion noise in a hybrid LES/APE-RF context. Note, the effect of unsteady heat release, which has been proven to be the most dominant acoustic source term in low-Mach number reacting flows, is inherently embedded within the two simplified source formulations. In this study, the following three formulations

$$
\begin{aligned}
q_{e, r f, S I A} & =-\bar{c}^{2} \frac{\bar{\rho}}{\rho} \frac{\gamma-1}{c^{2}} \sum_{n=1}^{N} h_{n} \omega_{n} \\
q_{e, r f, D \rho} & =-\bar{c}^{2} \frac{\bar{\rho}}{\rho} \frac{D \rho}{D t} \\
q_{e, r f, \partial \rho} & =-\bar{c}^{2} \frac{\partial \rho}{\partial t}
\end{aligned}
$$

are examined in terms of their numerical applicability to predict combustion noise in conjunction with the APE-RF system. Table (1) lists the source mechnisms inherently contained in these formulations.

Table 1. Source mechanisms contained in each simplified formulation of the RHS of the pressure density relation. The effects due to nonisomolar combustion, species diffusion, heat diffusion, and viscosity are denoted by $\sum_{i=2}^{N=5} q_{e, r f, i}$. The effect of the acceleration of density inhomogeneities is represented by $\nabla \cdot\left(u \rho_{e}\right)$.

\begin{tabular}{|c|c|c|c|c|c|}
\hline source & unst. heat release & $\sum_{i=2}^{N=5} q_{e, r f, i}$ & $\nabla \cdot\left(\boldsymbol{u} \rho_{e}\right)$ & $\boldsymbol{u} \cdot \nabla \bar{\rho}$ & pressure fluct. \\
\hline \hline$q_{e, r f, S I A}$ & $\times$ & - & - & - & - \\
\hline$q_{e, r f, D \rho}$ & $\times$ & $\times$ & - & - & - \\
\hline$q_{e, r f, \partial \rho}$ & $\times$ & $\times$ & $\times$ & $\times$ & - \\
\hline
\end{tabular}

\section{Numerical simulation}

\section{A. LES and flame configuration}

The large-eddy simulation of the so-called DLR-A flame has been performed using a low-Mach number variable density approach, while the combustion model is applied through the flamelet/progress variable (FPV) model by PIERCE \& MoIN. ${ }^{5,6}$ The DLR-A flame is an $\mathrm{N}_{2}$-diluted $\mathrm{CH}_{4}-\mathrm{H}_{2} /$ air flame, which has experimentally been investigated by Bergmann et al., ${ }^{7}$ Meier et al., ${ }^{8}$ and Schneider et al. ${ }^{9}$. For a detailed description of the LES, the flame specifications, and the modifications to the flamelet library to evaluate the thermoacoustic sources, the reader is referred to Bui et al. ${ }^{1}$.

\section{B. CAA domain and evaluation parameters}

The fine CAA domain is discretized using about $20 \times 10^{6}$ grid points covering $120 \mathrm{D}$ and $70 \mathrm{D}$ in the axial and the radial direction, respectively. The topology of the fine grid is chosen such that interpolation errors especially for the thermo-acoustic sources $\left(S_{I A}, S_{I B}, S_{I C}\right)$ are almost avoided. Since the distribution of the thermo-acoustic sources is closely linked to the surface of stoichiometric mixture, ${ }^{3}$ the source region is mainly divided into two areas. The grid point distribution in the outer area $(r / D \geq 0.5)$ coincides with the LES mesh, while the sources have to be interpolated in the inner area via a trilinear algorithm. 
The coarse mesh consists of about $18 \times 10^{6}$ grid points. These points, however, are distributed over a physical domain of $120 \mathrm{D}$ in the axial and $100 \mathrm{D}$ in the radial direction. The coarse mesh for the CAA simulation is able to resolve STROUHAL numbers up to $S t_{D}=1.89$ based on 5.4 points per wavelength (PPW) which can be achieved using the DRP scheme. The source terms are sampled with a time increment of $\Delta t_{s} c_{\infty} / D=0.3266$, which leads to a representation of the minimum time period of $T_{\min } / \Delta t=13.2$ points per period (PPP) at a maximum STROUHAL number of $S t_{D}=1.89$. Based on $5.4 \mathrm{PPW}$, the fine grid is able to resolve far more than $S t_{D}=2.0$. However, the time increment of the sampled source data limits the maximal resolvable frequency. A temporal resolution of 12.5 PPP is assumed to be sufficient at a maximum STROUHAL number of $S t_{D}=2.0$.

The CAA code is based on the fourth-order dispersion-relation preserving (DRP) scheme of TAM \& WEBB ${ }^{10}$ for the spatial discretization and the alternating low-dissipation low-dispersion RUNGE-KUTTA (LDDRK) method in the $5 / 6$ mode for the temporal integration by HU ET AL. ${ }^{11}$ to propagate the acoustic waves produced by the sources. A low-pass filtering method, i.e., a 6th-order explicit commutative filter ${ }^{12,13}$ is used at every fifth complete RUNGEKUTTA time step to remove spurious waves. A damping zone $e^{4,14}$ is introduced on the inner boundaries between the different matching blocks covering the LES and the acoustic domain to supress artificial noise due to a discontinuity in the entropy distribution. Since the APE system does not describe convection of entropy and vorticity modes, the asymptotic radiation boundary condition by TAM \& $\mathrm{WEBB}^{10}$ is used at far field boundaries to lower unphysical reflections into the computational domain.

\section{Results}

Three different sound source formulations have been studied in terms of their numerical applicability. These formulations differ from each other in their inherently contained source mechanisms. However, each model includes the effect of unsteady heat release rate. The impact of each source term will be investigated and compared with acoustic measurements by Singh et al. ${ }^{15}$.

\section{A. Source term analysis}

Figure 1 (left) shows the instantaneous source distribution of each formulation at two different axial positions, i.e., at $x / D=10$ and $x / D=30$, as a function of the radius. The corresponding wave number analysis is represented in the right diagrams of Figure 1. It is evident from Figure 1 that the partial time derivative shows the smoothest source distribution, which is characterized by a mainly low wave number content, while the wave number analysis of the source formulation $q_{e, r f, S I A}$, i.e., the unsteady heat release rate, illustrates a very sharp spatial distribution, which is characterized by a wave number content in a wide range. From this analysis it is certain that the interpolated source terms possess the smallest error for $q_{e, r f, \partial \rho}$, while the interpolation error for $S_{I A}$ should have a significant impact on the acoustic solution. Additionally, it can be expected from these findings that the interpolation error for the source formulation using the substantial time derivative of the density is lower than for $S_{I A}$. In brief, the formulation with the partial time derivative of the density is expected to reproduce the acoustic field best. However, it will be evidenced, that this is only true if a special constraint is fulfilled.

\section{B. Coarse grid vs. fine grid}

In Figures 3, 4, and 5, experimental and numerical results for different source formulations using a coarse and a fine grid are shown. The acoustic spectra are compared at five axial positions and a radial distance of $r / D=50$. A significant deviation in the higher frequency range starting from $S t_{D}>0.65$ can be observed between the coarse and the fine solution for the source formulations $q_{e, r f, D \rho}$ in Figure 4 and $q_{e, r f, S I A}$ in Figure 3. Due to the interpolation onto the coarse grid, this discrepancy has been expected and corresponds to a critical maximum wave number of $(k D)=1.54$ being resolved by the interpolation. Furthermore, the acoustic solution in Figure 3 excited by $q_{e, r f, S I A}$ on the coarse mesh also shows an almost constant deviation of $\Delta L_{p}=4 d B$ in the lower frequency range compared to the fine solution, which is in good agreement with the experimental data in the lower frequency range.

Analyzing the numerical findings of the partial time derivative of the density reveals an unexpected behavior. It has been shown before that this source term exhibits the smoothest source distribution, i.e., the impact of the interpolation error on the acoustic solution should be minimal. The results, however, show that besides the aforementioned deviation in the higher frequency range, which also occurs in the case of $q_{e, r f, \partial \rho}$, there are disparities in the lower frequency range, e.g., in Figures 5(a), (d) $\left(0.3 \leq S t_{D} \leq 0.65\right)$, which can not be explained by ordinary interpolation errors. A possible explanation for this unexpected behavior is given in the next subsection. 

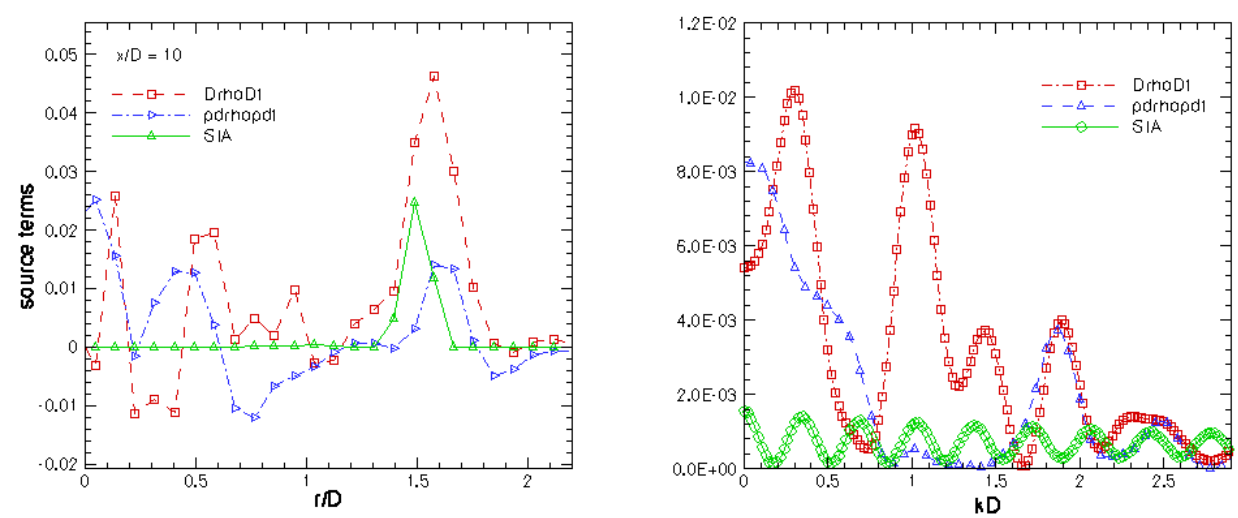

(a) $x / D=10$
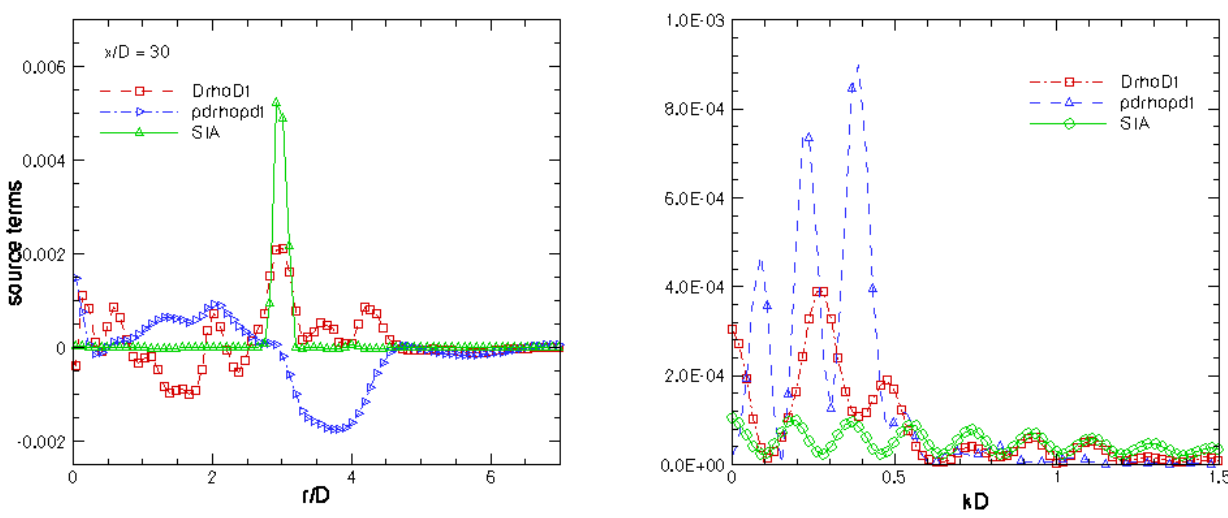

(b) $x / D=30$

Figure 1. Instantaneous dimensionless source distribution of different formulations in the radial direction at different axial positions (left) and associated wave number content in the radial direction (right). $q_{e, r f, D_{\rho}}=$ "DrhoDt", $q_{e, r f, \partial_{\rho}}=$ "pdrhopdt", and $q_{e, S I A}=" S I A "$

\section{Artificial acceleration due to interpolation}

What exactly is different between $q_{e, r f, D \rho}$ and $q_{e, r f, \partial \rho}$, which could lead to this unexpected behavior? To understand this problem, a numerical study is performed in which a GAUSSIAN density spot

$$
\rho(\boldsymbol{x}, t)=0.1 \exp \left(-\ln (2) \frac{\left(x-x_{0}(t)\right)^{2}+\left(y-y_{0}\right)^{2}}{0.5^{2}}\right)
$$

with

$$
x_{0}(t)=-9.0+\bar{u} t
$$

is constantly convected with a uniform mean flow $(\bar{\rho}, \bar{u}, \bar{v}, \bar{p})^{T}=(1,0.5,0,0.7142)^{T}$. The difference between the two formulations $q_{e, r f, \partial \rho}$ and $q_{e, r f, D \rho}$ is that the source term $q_{e, r f, \partial \rho}$ incorporates the effect of acceleration of density inhomogeneities. However, in this case, neither $q_{e, r f, D \rho}$ nor $q_{e, r f, \partial \rho}$ are supposed to excite acoustic responses, since an entropy spot, which is being convected constantly with the mean flow does not produce any noise. Apparently, this directly holds for $q_{e, r f, D \rho}$, since this source term vanishes by definition. Unlike $q_{e, r f, D \rho}$, the source term $q_{e, r f, \partial \rho}$ is non-zero for a constantly convecting entropy spot. Therefore, the influence of the source term interpolation on the acoustic field in investigated in the next step using $q_{e, r f, \partial \rho}$. 
For this test case, the convection of the density distribution in eqn. (11) is analytically prescribed with

$$
q_{e, r f}=-\bar{c}^{2} \frac{\partial \rho}{\partial t}
$$

on a fine mesh (Figure 2(a)). For the simulation on the coarse grid, the prescribed sources on the fine mesh are interpolated onto the coarser mesh (Figure 2(b)). Numerically, the entropy spot is initialized at $\boldsymbol{x}=(-9.0,0.0)$ without using an embedded inner damping zone, such that an initial acoustic response occurs. However, in the following, this entropy spot is just being convected at $\bar{u}$ and should therefore not produce any additional sound. In the analytically prescribed case in Figure 2(a) no acoustic disturbance is visible due to the convected spot. At this instant illustrated in Figure 2(a) the entropy spot has already been convected 15 units in the positive $x$-direction.

In the next step, the source terms of the first prescribed case are sampled with a time increment of $\Delta t=0.1$ and are interpolated onto the coarser grid, i.e., the same physical domain is discretized by $(112 \times 112)$ mesh points. Figure 2(c) shows the source position and the source contours at $t=30$. Since the mean flow speed in the $x$-direction has been set to be constant at $\bar{u}=0.5$, it appears that the entropy spot on the coarser grids is not only been convected, but obviously also been accelerated. The coarse grid has been chosen such that the mesh points do not coincide with the fine grid points. During interpolation of the GAUSSIAN density distribution it is obvious, that the maximum point of the GAUSSIAN distribution is permanently dislocated from its analytically prescribed position. A dipole-like sound field is been produced by this effect, which will be referred to as the "artificial interpolation induced acceleration" effect. The resulting sound field is shown in Figure 2(b). It is clearly demonstrated that this effect is able to generate significant spurious noise.

\section{Spectral content and directivity behavior}

As mentioned before, each source formulation defined in eqs. $(8,9,10)$ includes certain sound generating effects. A statement about the impact of some source mechanisms like $S_{I B}$ or $S_{I C}$ can be given implicitly when the spectral distributions in Figure 6 are compared.

It is evident from Figures 6 (a)-(d) that on the one hand, the acoustic impact in the lower frequency range $\left(S t_{D} \leq 0.45\right)$ is in general dominated by the effect of unsteady heat release rate, except at the location $(x / D, r / D)=$ $(50,50)$. Overall, this is in agreement with theoretical and experimental findings. ${ }^{16,17}$ On the other hand, this source effect appears to be insufficient to reproduce the acoustic field in the higher frequency range $\left(S t_{D}>0.45\right)$. Recall from Table 1 that the source formulation with the substantial time derivative $\left(q_{e, r f, D \rho}\right)$ includes additionally to the unsteady heat release rate, the effect of non-isomolar combustion, heat and species diffusion, and a term describing viscous heating. Comparing the spectra $q_{e, r f, D \rho}$ with those using $q_{e, r f, S I A}$ shows that the additional source mechanisms have an impact especially in the higher frequency range. In the downstream direction, i.e., at $(x / D, r / D)=(50,50)$, the spectrum is overpredicted in the frequency range $\left(S t_{D}>0.8\right)$, while at the observer position close to the jet exit $(x / D, r / D)=(0,50)$, those additional sources are still not able to compensate the lack between the acoustic response caused by the unsteady heat release rate and the experimental data. However, the acoustic field excited by $\left(q_{e, r f, D \rho}\right)$ shows a good agreement in a wide frequency range at almost all observer locations.

During the derivation of the simplified source formulation $q_{e, r f, \partial \rho}$ only one simplification has been applied to the RHS of the pressure-density relation, i.e., pressure fluctuations have been neglected. Hence, as also listed in Table (1), this formulation includes the most sound source effects. Compared with the other formulations, the analysis of Figure 6 shows at each observer location a very good agreement of the numerical results using $q_{e, r f, \partial \rho}$ with the experimental data. The numerical results, especially in Figure 6(a) suggest the acoustic response from the extra sources, expressed through the difference between the two formulations $\left(q_{e, r f, \partial \rho}-q_{e, r f, D \rho}\right)$, namely the effect of the acceleration of density inhomogeneities and the effect of mean density gradients, to lie in the higher frequency band and to possess a preferred radiation direction of $90^{\circ}$ to the flame axis close to the jet exit. A significant contribution of

the thermoacoustic sources $\sum_{i=2}^{N=5} q_{e, r f, i}$ to the acoustic field can be observed mainly in the higher frequency range $\left(S t_{D}>0.45\right)$ at each observer position. Moreover, the spectra in Figures 6(d),(e) reveal an increasing impact of the thermoacoustic sources in the lower frequency range at downstream monitor points, for instance at $x / D=50$.

\section{Conclusions}

Three different simplified formulations of the RHS of the pressure-density relation have been used to simulate the acoustic field generated by the DLR-A flame, a non-premixed turbulent flame. To take advantage of the scale separation in aeracoustics, a hybrid solution approach has been used. That is, the source terms of the APE-RF system 


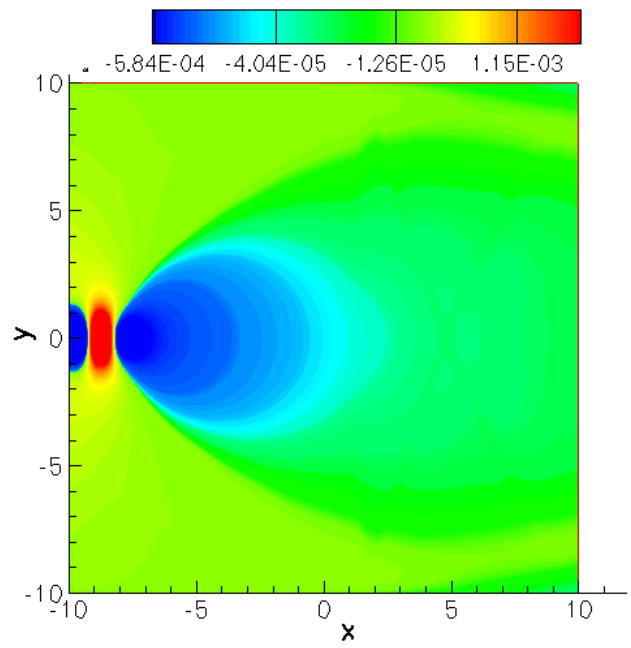

(a) $401 \times 401$ grid points

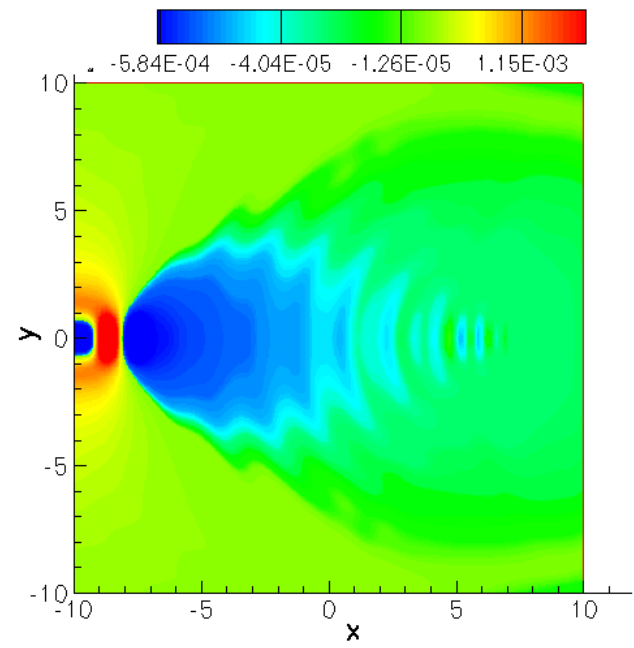

(b) $112 \times 112$ grid points

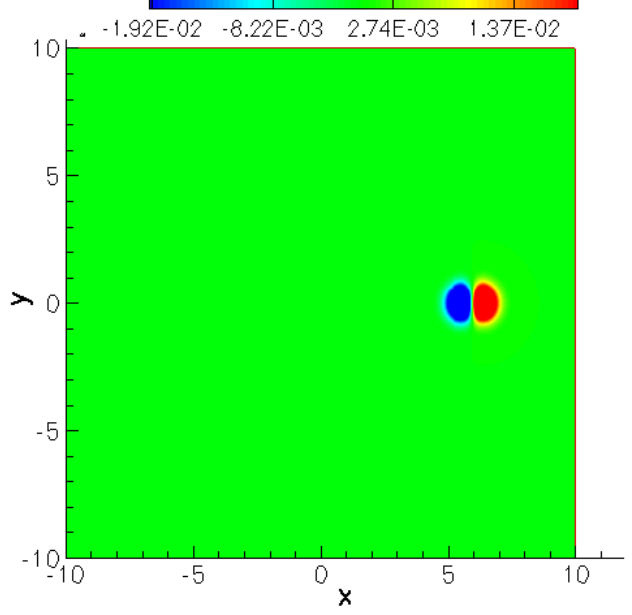

(c) $q_{e, r f}$ at $t=30$

Figure 2. Instantaneous perturbation pressure $p^{\prime}[-]$ contours of an entropy spot at $t=30$ convecting with a mean flow speed of $\bar{u}=0.5$ in the $x$-direction, on three meshes with different resolutions $(\mathbf{a}, \mathbf{b})$. At this moment, the entropy spot has been convected to the position $\boldsymbol{x}_{0}=(6,0)(\mathbf{c})$. 
have been evaluated on the LES mesh and afterwards mapped onto the CAA grid to propagate the sound waves. The acoustic results for the non-premixed turbulent flame can be summarized in three categories.

Firstly, the results obtained on the coarse grid using the three source formulations are compared with each other. Due to interpolation errors the acoustic responses of the sources $q_{e, r f, S I A}$ and $q_{e, r f, D \rho}$ show deviations from the experimental data in a frequency range $S t_{D} \geq 0.65$. The acoustic response due to the interpolated unsteady heat release source on the coarse grid is characterized by an almost constant drop of $\Delta L_{p}=4 d B$. Though the source expression $q_{e, r f, \partial \rho}$ exhibits the smoothest source distribution, the acoustic response on the coarse grid also shows deviations in the frequency range $\left(0.3 \leq S t_{D} \leq 0.65\right)$, which cannot be explained by ordinary interpolation errors.

Secondly, the "artificial interpolation induced acceleration" effect has been shown to considerably falsify the acoustic solution in conjunction with $q_{e, r f, \partial \rho}$. The unexpected aforementioned acoustic response of the source $q_{e, r f, \partial \rho}$ on the coarse grid can at least be partially attributed to this effect. The use of this source term requires preserving the convection speed of density inhomogeneities during interpolation.

Thirdly, the following conclusions about the spectral content and directivity behavior of the different source mechanisms may be drawn directly or implicitly from the results obtained on the fine grid. The effect of unsteady heat release dominates the acoustic response in the lower frequency range, $S t_{D} \leq 0.45$, except at the observer location $(x / D, r / D)=(50,50)$. However, the use of this source is insufficient to reproduce the acoustic field in the higher frequency range $S t_{D}>0.45$. A statement on the impact of the source mechanisms $\left(q_{e, r f, D \rho}-q_{e, r f, S I A}\right)$, i.e., the effect of non-isomolar combustion, species and heat diffusion, can be given implicitly when the results obtained from $q_{e, r f, S I A}$ and $q_{e, r f, D \rho}$ are compared. These source mechanisms have an effect on the higher frequency range. Furthermore, the acoustic impact is even evident in the lower frequency range at the downstream observer position. The difference between $q_{e, r f, \partial \rho}$ and $q_{e, r f, D \rho}$ represents the effect of acceleration of density inhomogeneities and mean density gradients. These mechanisms produce an acoustic output with a preferred radiation direction of $90^{\circ}$ to the flame axis.

In terms of numerical applicability of these source formulations it can be concluded that the source term $q_{e, r f, S I A}$ is inappropriate to predict the acoustic field of reacting flows for two reasons. The evaluation of this source term requires additional effort and the use of this term leads to a very stiff problem.

The comparison of the source terms shows the substantial time derivative of the density to be a good compromise between numerical accuracy and efficiency. Moreover, this formulation does not suffer from the "artificial interpolation induced acceleration" effect. However, if the convection speed can be preserved during interpolation, the formulation using the partial time derivative of the density is to be preferred.

\section{Acknowledgements}

The financial support by the German Research Foundation (DFG) through the Research Unit FOR 486 "Combustion Noise" is gratefully acknowledged.

\section{References}

\footnotetext{
${ }^{1}$ Bui, T. P., Ihme, M., Meinke, M., Schröder, W., and Pitsch, H., "Numerical Investigation of Combustion Noise and Sound Source Mechanisms in a Non-premixed Flame using LES and APE-RF," AIAA Paper 2007-3406, 2007.

${ }^{2}$ Ewert, R. and Schröder, W., "Acoustic perturbation equations based on flow decomposition via source filtering," J. Comput. Physics, Vol. 188, 2003, pp. 365-398.

${ }^{3}$ Bui, T. P., Schröder, W., and Meinke, M., "Acoustic perturbation equations for reacting flows to compute combustion noise," Int. J. Aeroacoustics, Vol. 6, No. 4, 2007, pp. 335-355.

${ }^{4}$ Bui, T. P., Meinke, M., and Schröder, W., "Numerical analysis of the acoustic field of reacting flows via acoustic perturbation equations," Comput. \& Fluids (accepted for publication), 2007.

${ }^{5}$ Pierce, C. and Moin, P., "Progress-variable approach for large-eddy simulation of non-premixed turbulent combustion," J. Fluid Mech., Vol. 504, 2004, pp. 73-97.

${ }^{6}$ Ihme, M., Cha, C. M., and Pitsch, H., "Prediction of local extinction and re-ignition effects in non-premixed turbulent combustion using a flamelet/progress variable approach," Proc. Combust. Inst., Vol. 30, 2005, pp. 793-800.

${ }^{7}$ Bergmann, V., Meier, W., Wolff, D., and Stricker, W., "Application of spontaneous Raman and Rayleigh scattering and 2D LIF for the characterization of a turbulent $\mathrm{CH}_{4} / \mathrm{H}_{2} / \mathrm{N}_{2}$ jet diffusion flame," Appl. Phys. B, Vol. 66, No. 4, 1998, pp. 489-502.

${ }^{8}$ Meier, W., Barlow, R., Chen, Y., and Chen, J., "Raman/Rayleigh/LIF measurements in a turbulent $\mathrm{CH}_{4} / \mathrm{H}_{2} / \mathrm{N}_{2}$ jet diffusion flame: Experimental techniques and turbulence-chemistry interaction," Combust. Flame, Vol. 123, No. 3, 2000, pp. 326-343.

${ }^{9}$ Schneider, C., Dreizler, A., Janicka, J., and Hassel, E., "Flow field measurements of stable and locally extinguishing hydrocarbon-fuelled jet flames," Combust. Flame, Vol. 135, No. 1-2, 2003, pp. 185-190.

${ }^{10}$ Tam, C. K. W. and Webb, J. C., "Dispersion-Relation-Preserving Finite Difference Schemes for Computational Acoustics," J. Comput. Physics, Vol. 107, No. 2, 1993, pp. 262-181.
} 
${ }^{11}$ Hu, F. Q., Hussaini, M. Y., and Manthey, J. L., "Low-Dissipation and Low-Dispersion Runge-Kutta Schemes for Computational Acoustics," J. Comput. Physics, Vol. 124, No. 1, 1996, pp. 177-191.

${ }^{12}$ Shang, J. S., "High-order compact-difference schemes for time dependent maxwell equations," J. Comput. Physics, Vol. 153, 1999 , pp. 312 -333 .

${ }^{13}$ Vasilyev, O. V., Lund, T. S., and Moin, P., "A general class of commutative filters for LES in complex geometries," J. Comput. Physics, Vol. 146, 1998, pp. $82-104$.

${ }^{14}$ Schröder, W. and Ewert, R., "LES-CAA Coupling, LES for Acoustics", Cambridge University Press, 2005.

${ }^{15}$ Singh, K., Frankel, S., and Gore, J., "Study of Spectral Noise Emissions from Standard Turbulent Nonpremixed Flames," AIAA Journal, Vol. 42, No. 5, 2004.

${ }^{16}$ Smith, T. J. B. and Kilham, J. K., "Noise Generation by Open Turbulent Flames," J. Acoust. Soc. America, Vol. 35, No. 5, 1963 , pp. 715 724.

${ }^{17}$ Strahle, W. C., "Some results in combustion generated noise," J. Sound Vibration, Vol. 23, No. 1, 1972, pp. 113 - 125. 


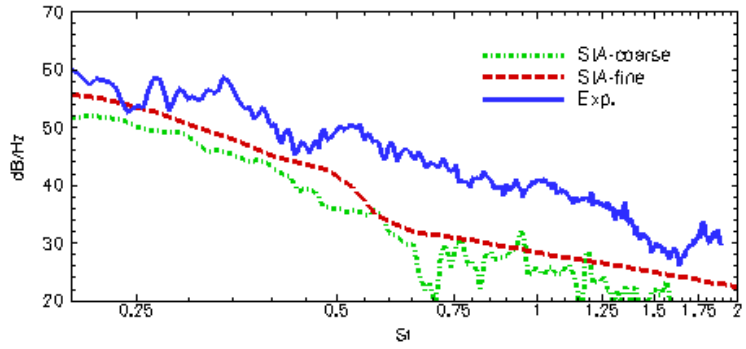

(a) $(x / D, r / D)=(0,50)$

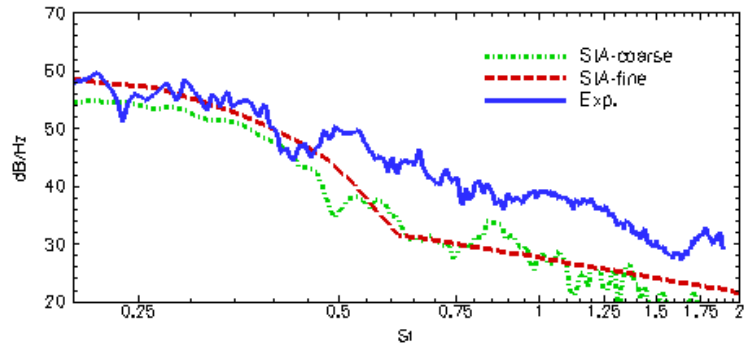

(b) $(x / D, r / D)=(12,50)$

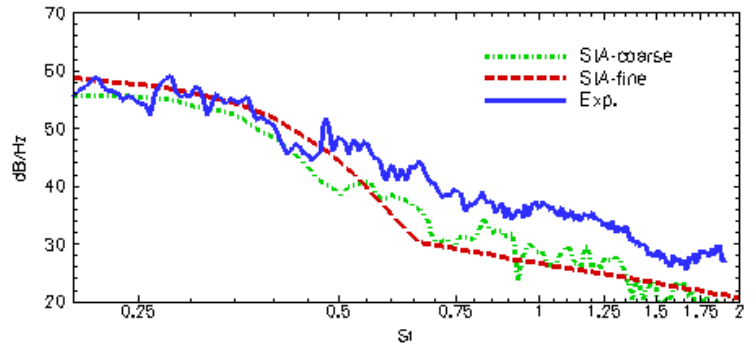

(c) $(x / D, r / D)=(25,50)$

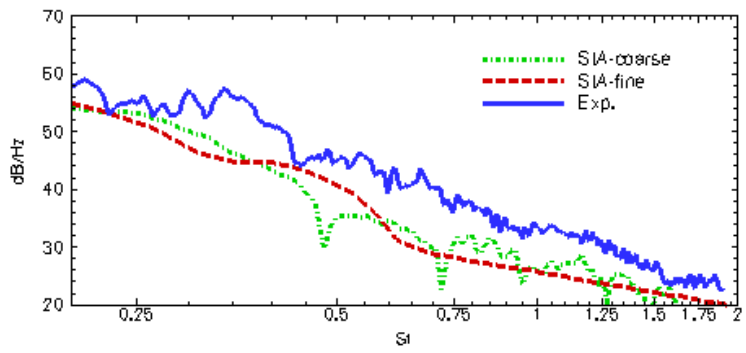

(d) $(x / D, r / D)=(37,50)$

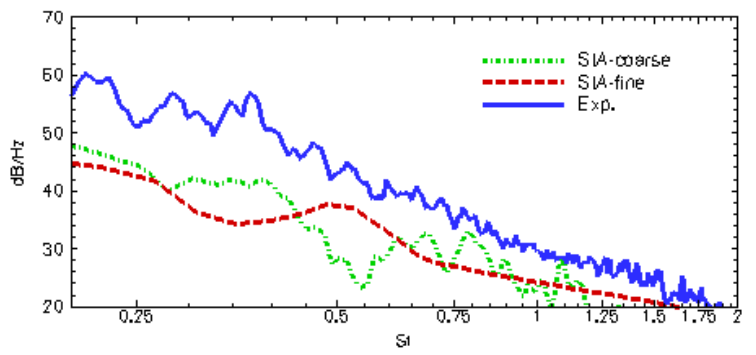

(e) $(x / D, r / D)=(50,50)$

Figure 3. Spectra in the frequency range of $0.2 \leq S t_{D} \leq 2$ are shown. Comparison of computed sound pressure levels on a coarse and a fine grid at $r / D=50$ and five different axial $x / D$ locations using $q_{e, r f, S I A}$. The STROUHAL number is based on the jet exit velocity and the nozzle diameter $D$. 


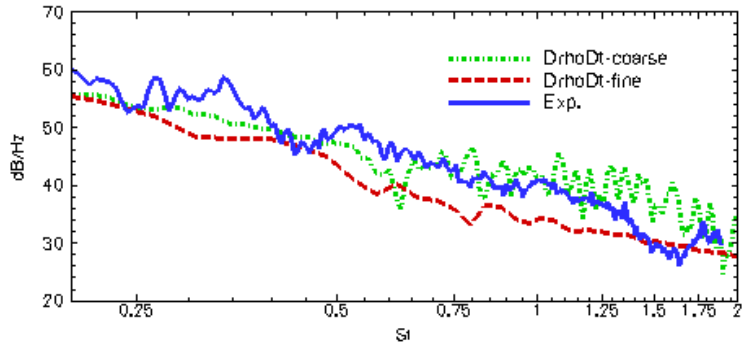

(a) $(x / D, r / D)=(0,50)$

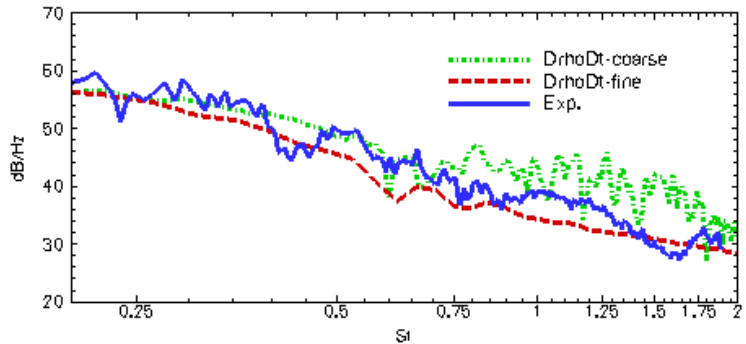

(b) $(x / D, r / D)=(12,50)$

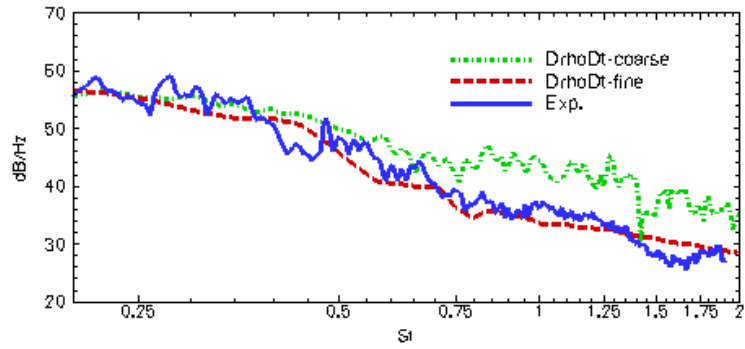

(c) $(x / D, r / D)=(25,50)$

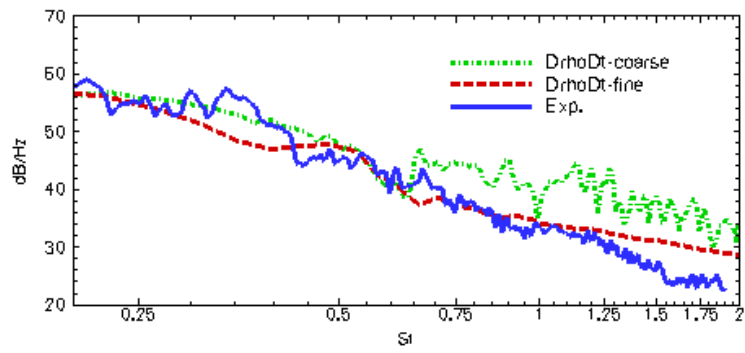

(d) $(x / D, r / D)=(37,50)$

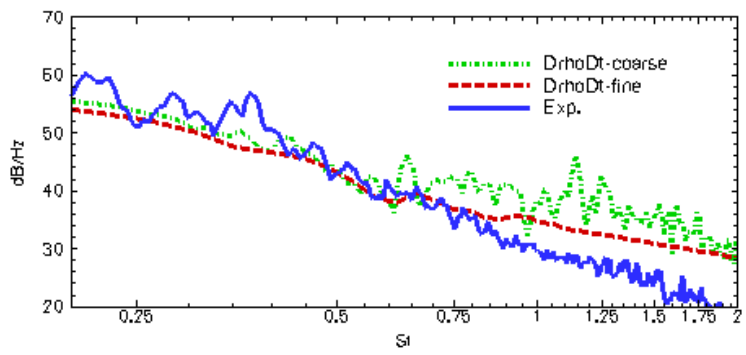

(e) $(x / D, r / D)=(50,50)$

Figure 4. Spectra in the frequency range of $0.2 \leq S t_{D} \leq 2$ are shown. Comparison of computed sound pressure levels on a coarse and a fine grid at $r / D=50$ and five different axial $x / D$ locations using $q_{e, r f, D_{\rho}}$ (right). The STROUHAL number is based on the jet exit velocity and the nozzle diameter $D$. 


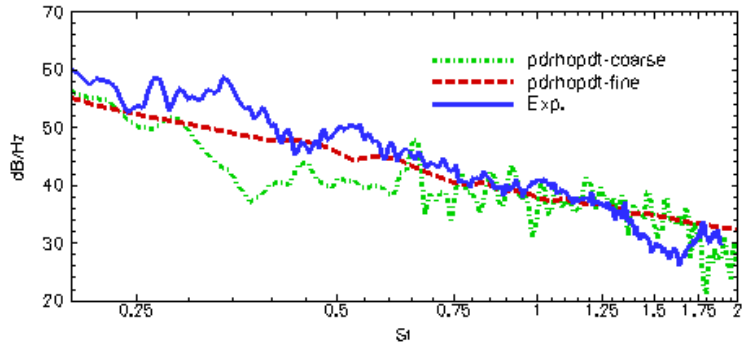

(a) $(x / D, r / D)=(0,50)$

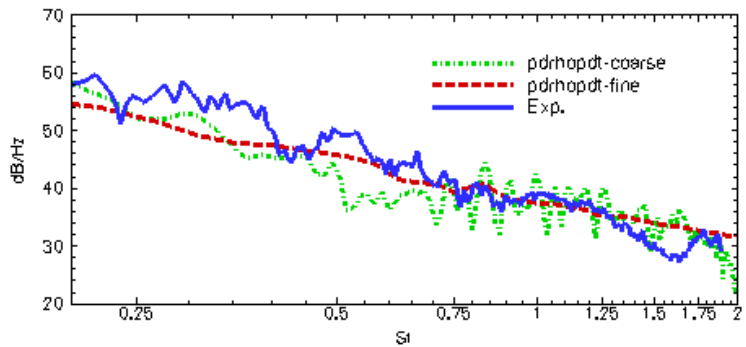

(b) $(x / D, r / D)=(12,50)$

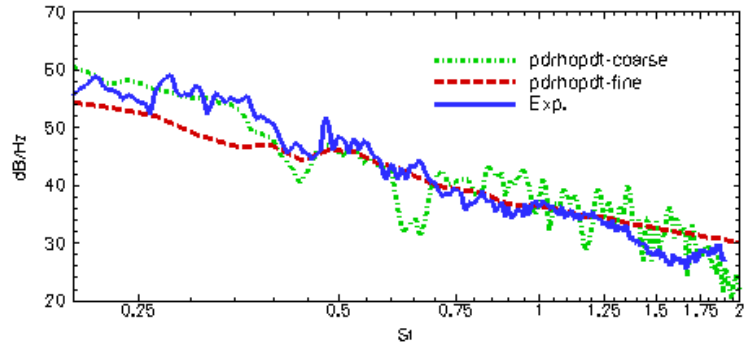

(c) $(x / D, r / D)=(25,50)$

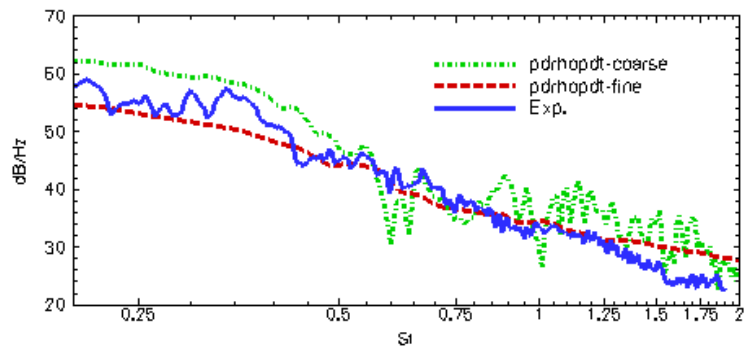

(d) $(x / D, r / D)=(37,50)$

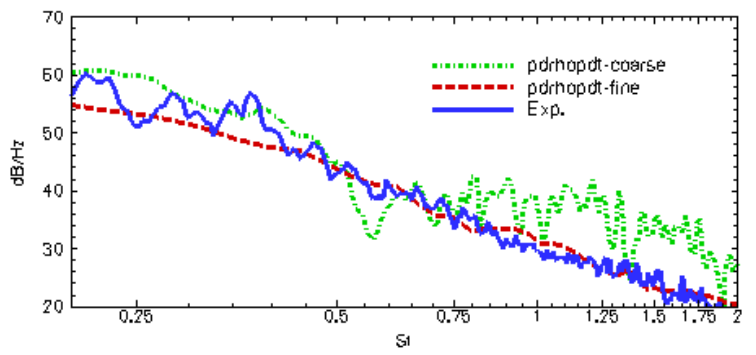

(e) $(x / D, r / D)=(50,50)$

Figure 5. Spectra in the frequency range of $0.2 \leq S t_{D} \leq 2$ are shown. Comparison of computed sound pressure levels on a coarse and a fine grid at $r / D=50$ and five different axial $x / D$ locations using $q_{e, r f, \partial_{\rho}}$. The STROUHAL number is based on the jet exit velocity and the nozzle diameter $D$. 


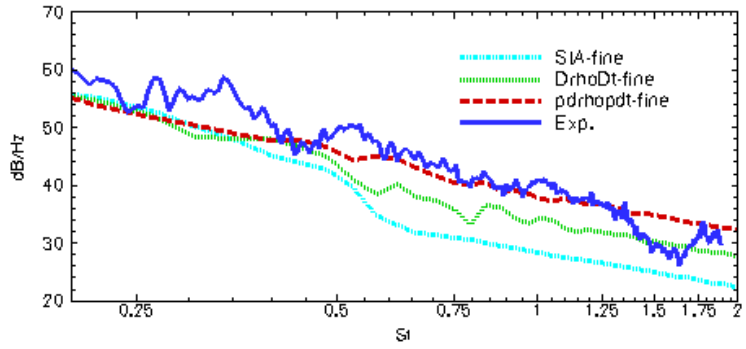

(a) $(x / D, r / D)=(0,50)$

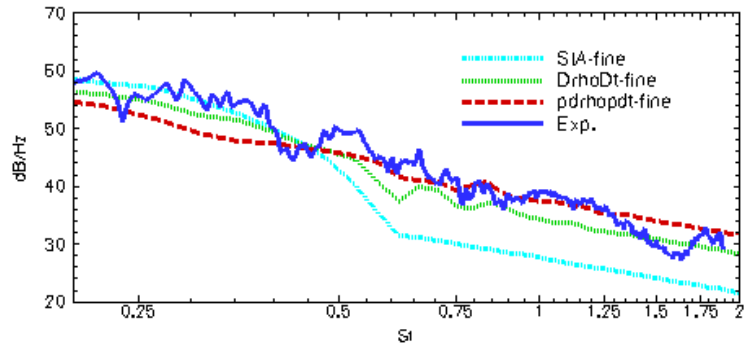

(b) $(x / D, r / D)=(12,50)$

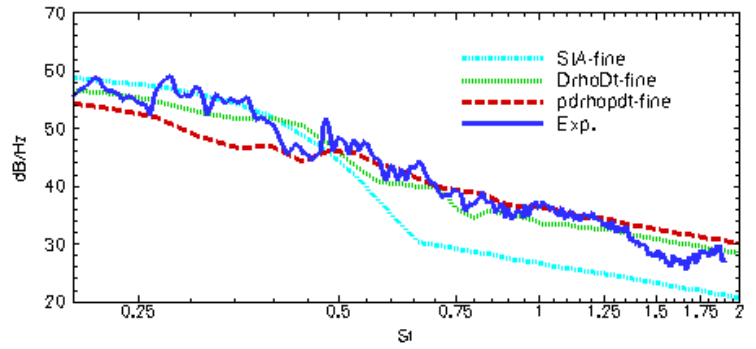

(c) $(x / D, r / D)=(25,50)$

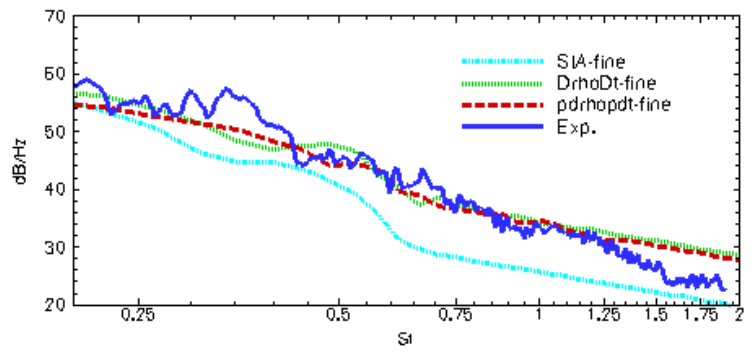

(d) $(x / D, r / D)=(37,50)$

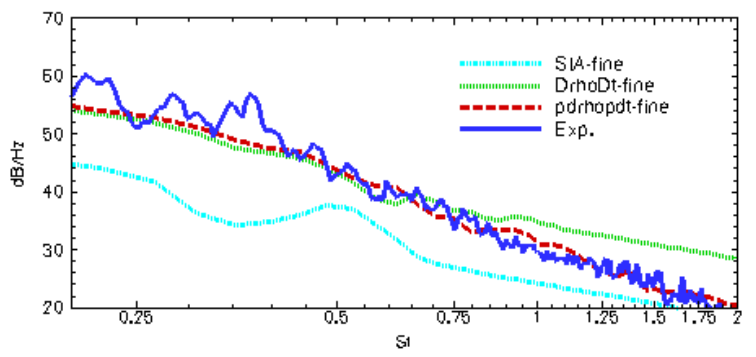

(e) $(x / D, r / D)=(50,50)$

Figure 6. Spectra in the frequency range of $0.2 \leq S t_{D} \leq 2$ are shown. Comparison of measured and calculated sound pressure level at $r / D=50$ and five different axial $x / D$ locations using different sound source formulations. The STROUHAL number is based on the jet exit velocity and the nozzle diameter $D$. 